\title{
A novel personal ventilation strategy to circumvent aerosol transmission
}

\section{Tomoaki Yoshida ( $\nabla$ tomo-y@fujita-hu.ac.jp )}

Fujita Health University

\section{Eiji Shibata}

Aichi Medical University

\section{Natsuki Ishida}

Seto Health Check Center

\section{Tomoya Nakatani}

Seto Health Check Center

\section{Research Article}

Keywords: SARS-cov2, aerosol transmission, intervention, zoning, ventilation

Posted Date: March 15th, 2021

DOI: https://doi.org/10.21203/rs.3.rs-215168/v1

License: (c) (1) This work is licensed under a Creative Commons Attribution 4.0 International License. Read Full License 


\section{Abstract}

In the pandemic of COVID-19, a rigorous lockdown or social distancing will mitigate transmission, but some alternative strategy is needed especially for mass-gathering events. Given that the main path of transmission is through droplets or aerosols, a swift removal of them immediately after exhalation, which may attain "personal air zoning", would be more effective and feasible than whole room ventilations. In the present study, an artificial fog was employed as a model aerosol to be exhaled and readily visualized on movies and quantified on dust indicators. The temporal and spatial distribution of this model microdroplet amounts corresponded reasonably well with previously published data, where talking air flow was quantified as a negative staining, in that it predominates below the mouth height rather than horizontal, and that it travels forward over $1.5 \mathrm{~m}$ in $30 \mathrm{sec}$. Under this model condition, nearly $99 \%$ of exhaled microdroplets could be efficiently blown up beyond the bystanders' head heights, when a minimal air flow $(2.5 \mathrm{~m} / \mathrm{s})$ was applied, using a typical personal cooling fan just below the chin. This swift upward removal of microdroplets would prevent bystanders' immediate inhalation and provide sufficient probation periods for safe exhaustion from indoor spaces.

\section{Introduction}

A number of superspreading events have been observed throughout the pandemic of COVID-19 ${ }^{12}$ and would, at least in part, account for the huge wave of outbreak in the world ${ }^{3,4}$. Indeed, the dispersion coefficient, which negatively correlates with the numbers of offspring patients, ranges 0.05-0.4 depending on studies ${ }^{5}{ }^{8}$, which are comparable with SARS $(0.16){ }^{9}$, and indicates contributions of superemitting patients ${ }^{10}$. Among those superspreading cases, some common features of circumstances are apparently shared, such as closed indoor areas, poor ventilations, and crowded people ${ }^{1}$.

During the early epidemic in China, cases in a restaurant and a bus tour were reported in relevant conditions ${ }^{11} 12$. So was a Church cluster of 112 patients, out of approximately 150 attendants, in Bremerhaven Germany in June ${ }^{13}$, and was a recent cluster of 61 patients among musical actors and staffs in Saitama Japan ${ }^{14}$. In the last case, notably, actors and staffs proclaimed to have pursued thorough disinfection of high-touch surfaces, and worn face mask or mouth shield. On the contrary, just a few cases of small-scale transmissions were observed in outdoor conversations ${ }^{1}$.

A deliberate consideration on these superspreading phenomena, would rather prefer aerosol-mediated transmission ${ }^{15}$ to conventional contact or large droplet-mediated ones ${ }^{16}$. A mathematical estimation that only $10-20 \%$ were supposed to transmit to $80 \%$ of the whole patients ${ }^{17}$, would further support the accountability of aerosol infection for lots of large cluster infections.

Indeed, a recent study has revealed that some patients expel up to over $10^{5}$ virions per min just by breathing, and surprisingly less than $10 \%$ of surface swabs from mobile phones found positive ${ }^{18 \_20}$. The outrageous number of SARS-cov2 virions emitted by breathing and coughing of superemitters ${ }^{21}$ might 
surpass those in influenza ${ }^{22}$. In addition, the SARS-cov2 appeared to survive with half-life of $1.1 \mathrm{~h}$ in aerosol ${ }^{23}$, which is more than two-times longer than influenza ${ }^{24}$. Although a recent mathematical model is suggesting a few thousand vial copies as the minimal infectious load ${ }^{25}$, the present pandemic might be attributable to such tough viral characters.

Many cases of superspreading events have some relevance with talking or singing, which produce far beyond 10-times more droplets than just breathing. The degree of increment by talking has been reported to correlate with the square root of loudness, but there are some "speech superemitters" who release nearly 3-times more of an average person ${ }^{26}$. Compared with talking, singing has been reported to produce approximately 6 -times more droplets ${ }^{27}$. The average droplet size emitted by talking became slightly larger than just breathing, presumably reflecting the participation of laryngeal fluid film bursts ${ }^{26}$. In fact, it has already been argued that the droplets would have originated from 3 distinct anatomical areas of respiratory tract, lung, larynx, and oral cavity, thus, depict 3 peaks in the size distribution ${ }^{28}$.

Although the largest ones, probably from oral cavity, may surpass $100 \mu \mathrm{m}$ diameter and settle down in several seconds ${ }^{29}$, the other ones of smaller than $80 \mu \mathrm{m} \varphi$ evaporate in less than $8 \mathrm{~s}$ at $50 \%$ humidity and would remain in air for one min to over one day depending on their sizes. For example, even a $10 \mu \mathrm{m} \varphi$ droplet, that is twice as large as the border of "large droplet", sediments $2 \mathrm{~m}$ in $11 \mathrm{~min}$ as such, but it does in 154 min along with evaporation at $50 \%$ humidity ${ }^{30}$. Here, we need to recall that SARS-cov2 readily infects oral epithelial cells as well as nasopharyngeal ones, due to its usage of ACE2 as the receptor ${ }^{31}$, thus, remarkable numbers of virions might be speculated in larger droplets from oral cavity ${ }^{32}{ }^{33}$. This assumption may contrast with influenza, which is detectable predominantly in the nasopharyngeal region as RNA ${ }^{34}$ and contained more in smaller microdroplets 22,35 .

To avoid the spread of infectious droplets in air, we should first consider the air flow dynamics of speaking ${ }^{36}$. The flow speed is reported to range $0.1-1.5 \mathrm{~m} / \mathrm{s}$, at the fastest with plosive consonants, and could reach beyond $2 \mathrm{~m}$ forward ${ }^{37}$ through making "a train of air puffs" in less than $20 \mathrm{~s}$, just as a dilution of about $3 \%{ }^{38}$.

Those background facts that the huge numbers of infectious droplets could diffuse from a speaking or singing patient for significant distances and durations, would further support the relevance of aerosol transmission in closed indoor areas. A classical idea of intervention with aerosol transmission is to ventilate the indoor air intermittently or continuously, allowing a significant energy loss for temperature control. An alternative strategy to reduce energy loss may be to build in so-called personal ventilation equipment, somewhere around chairs or desks ${ }^{39}$.

Here, we would like to propose a practical intervention through removing aerosol right after exhalation employing a simple personal device of fan, instead of aiming at total air exchange. Since the device employed is placed just below the chin, just a minimal air speed can remove the exhaled droplets from the mouth height, so that surrounding subjects could hardly inhale them. 


\section{Results}

The droplets used here represent air-borne microdroplets but not larger ones

The droplet size distribution of the fog machine appeared $0.5-4 \mu \mathrm{m} \varphi$, when being deposited on a plastic dish and examined under a phase-contrast microscope (supplementary fig. S1). This size range would represent smaller microdroplets or aerosol. Thus, videos of exhaled fog have visualized probable behaviors of air-born microdroplets, but not larger ones that would be expelled rather straight frontward. The relative amounts of microdroplets were quantified as cpm (count per a minute) on digital dust meters.

The microdroplets were exhaled predominantly downward and travelled over $1.5 \mathrm{~m}$.

The videos of smoke exhalation have depicted remarkable downward directions, as being represented by about $40^{\circ}$ with voluntary coughing (supplementary fig. S2). In addition, the nasal exhalation, for example, after saying "Stay", seemed nearly $70^{\circ}$ downward and would be contributing to the overall direction further. Indeed, the relative amounts of oral and nasal exhalation while saying "Stay healthy" was found almost equivalent, and were even reverse while pronouncing " $n$ ", as shown in Fig. 1a. Accordingly, more microdroplets distributed $0.5 \mathrm{~m}$ below the mouth level, at $0.5 \mathrm{~m}$ frontward (Fig. $1 \mathrm{~b}, \mathrm{P}=0.007$ ). Interestingly, droplets were not significantly detected at $1 \mathrm{~m}$ below, through $0.5 \mathrm{~m}(\mathrm{P}=0.17)$ to $1.5 \mathrm{~m}$ frontward. On the other hand, droplets were sequentially observed $0.5 \mathrm{~m}$ below while counting the average cpm for $10 \mathrm{sec}$ and $30 \mathrm{sec}$ (Fig. 2), which indicates an almost horizontal movement at that height. These results of droplet distribution corresponded with a recent study, where a "negative staining" on a fog background was employed ${ }^{38}$, and would confirm the reliability of the present data as a model simulation experiment.

A slow-speed fan just below the chin blows up most of exhaled microdroplets

A common personal cooling device for Summer provides approximately $2.5 \mathrm{~m} / \mathrm{s}$ air flow at $10 \mathrm{~cm}$, which corelates with a typical distance to the mouth. Since the air flow of talking ranges $0.1-1.5 \mathrm{~m} / \mathrm{s}$, this slow flow speed was fast enough to efficiently blow the microdroplets upward and make the frontward undetectable (Fig. 3, $\mathrm{P}=0.009$ ). The usage of mouth shield has further improved the efficiency (Fig. 3, $\mathrm{P}<0.001$ ) and the direction of resulting air flow seemed almost vertically upward on video (Supplementary video). Although the video could not visualize the whole path of microdroplets, probably due to its fast motion, the dust meter has detected them a few centimeters away around the face and ears. Accordingly, the still picture (supplementary Fig. S3) depicted predominantly confined fog inside the mouth shield.

The height reached under these conditions with the fan was proved to exceed $0.9 \mathrm{~m}$ as shown in Fig. 4 $(P<0.001)$, thus, it would almost protect bystanders from inhaling the contaminated aerosol for a while. This "probation" period could be approximately $20 \mathrm{~min}$, based on the theoretical falling time of $5 \mu \mathrm{m} \varphi$ droplet at $100 \%$ humidity ${ }^{30}$. The aerosol could be safely removed through exhausting system, depending on the intrinsic ability of building ventilations. 


\section{Discussion}

Here, we would propose a novel strategy to circumvent exposures to bystander persons' exhaled microdroplets in closed spaces. The large droplets, however, must be trapped with a mouth shield first, and might be evaporated to become microdroplets. A simple small fan below the chin could blow nearly $99 \%$ of microdroplets upward from persons' head heights (Figure 3,4 ), allowing undetectable amounts frontward (supplementary Fig. S3 and supplementary video). Once microdroplets are removed for "probation", they could be exhausted within the sedimentation times 30 . On the contrary, the exhaled microdroplets, which moves predominantly downward (Figure 1b), would usually stay at or below people's head heights 38,40 , since vertical movements of air might be negligible with least air flow. Indeed, the microdroplets were hardly detected at $0.3 \mathrm{~m}$ above head in $30 \mathrm{sec}$ (Figure 4, $\mathrm{P}=0.40$ ), whereas were significantly detected at $1 \mathrm{~m}$ frontward in $30 \mathrm{sec}$ (Figure 2, P=0.012), which might be reflecting predominantly horizontal axis of humane activities. This reservation of aerosol around a patient would probably contribute to distant transmissions in a closed room after a long duration. The microdroplet seems to travel slower (Figure 2) than a previous data obtained with a "negative staining" on a fog 38. This discrepancy may be, in part, attributable to the huge mass size differences, between water droplets $(\mu \mathrm{m})$ and air molecules (sub $\mathrm{nm}$ ). Even though the classical recommendation of whole room ventilations has been introduced globally, a thorough indoor air exchange would result in loss of energy and sometimes be tough depending on the structures of buildings. In fact, the stringencies of legal regulations or guidelines on ventilation equipment and systems widely differ across nations. Especially, some fundamental policies seem quite distinct between western countries and eastern Asia, presumably due to climate and cultural differences. Many European countries rather demand airtightness legally 41, but not does the Japanese building standard law 42. On the contrary, only about half of European countries require certain ventilation standards, and even the required demands are mostly less strict than Japan 41,42 . The Japanese regulation in 2003 has been requiring all the new office buildings to be equipped with machinery ventilations ( $20 \mathrm{~m} 3 / \mathrm{h}$ •each person's space: i.e., $3 \mathrm{~m} 2$ for restaurants) and to be substituted to regular inspections. In addition, public halls or video theaters need to ventilate with fresh air at $75 \mathrm{m3} / \mathrm{h} \cdot \mathrm{m} 2$. Concerning old buildings of schools (not universities) and churches, in Japan, it is of note that they are installed with numbers of additional upper windows above the ordinary ones for swift ventilation. We ought to recall the impressive fact that no apparent cluster infection of COVID-19 has been announced in Japanese churches 43. On the other hand, there have been a great number of cluster cases in karaoke bars or cafes in Japan, many of which were poorly ventilated. A recent superspreading case in Dutch nursing home is also attributable to an inadequate ventilation that automatically recirculated the indoor air to save energy 16 . To highlight the crucial effects of ventilation further, we should present an inspiring fact that another karaoke business style, so-called "Karaoke boxes" in Japan 44 , where quite superior machinery ventilations are equipped $(20-30 \mathrm{~m} 3 / \mathrm{m} 2 / \mathrm{h})$, has not afforded any apparent cluster cases, even though they have been attended by over ten million people since the last June. Our preliminary smoke test has shown that vertical air flows from the ceiling seems to predominate in their rather narrow rooms and might be functioning as a sort of "air zoning" 45 . The fundamental idea of the present strategy is to remove each person's breath, immediately after exhaled, toward the overhead 
zones with a minimal upward air flow. When we could apply this strategy to each participant in a closed indoor space, even a poor ventilation machine would be able to handle the possible contaminated air much more efficiently, without rudimentary renovation of the building. Moreover, an additional weak horizontal air flow (ca. $3 \mathrm{~m} / \mathrm{sec}$ ), at the overhead zone, could move the microdroplets to an intended direction, at least $4 \mathrm{~m}$ away from the index person (our preliminarily data). Such combinations of simple air flows would efficiently reduce the risk of microdroplet inhalations and might eventually mitigate superspreading events, especially when talking, singing or having dinner parties. In conclusion, our simulation model experiments have shown that most of microdroplets exhaled could be removed swiftly by a slow air flow of about $2.5 \mathrm{~m} / \mathrm{s}$ below the chin. The efficiency of removal seemed nearly $99 \%$, and up above $0.9 \mathrm{~m}$ high, where falling time would be longer than $10 \mathrm{~min}$. A subsequent combination of built-in indoor ventilation flow, and possibly, supplemental horizontal air flows, would exhaust the removed droplets overhead, to attain a practical "Air Zoning" or "Invisible Exhaust Duct".

\section{Methods}

The participant

The participant in this study is the corresponding author alone and provided the consent for publication. This study, therefore, fulfills relevant guidelines and regulations.

The model microdroplets and their quantification

The aerosol behavior, while talking, was visualized and quantified by inhaling droplets from a fog machine, Z800II (Antari Lighting \& Effects Ltd., Taoyuan City 338, Taiwan) and by saying "Stay Healthy" repeatedly. The fog liquid used here, FLG-5 (Antari Lighting \& Effects Ltd.), was a long-lasting type and remained visible for at least $30 \mathrm{~min}$. The amounts of droplets exhaled were assessed by a digital dust indicator (LD-5R, Shibata Scientific Technology Ltd., Saitama, Japan), which measures laser light scattering as aspirating at $1.7 \mathrm{~L} / \mathrm{min}$. The data correlates with total mass concentration of droplets $(0.1$ $100 \mu \mathrm{m} \varphi$ ) without distinguishing the size. The droplet amounts were accumulated and averaged for 10 sec except described otherwise. One count per $\min (\mathrm{cpm})$ represents $0.001 \mathrm{mg} / \mathrm{m}^{3}$ of standard particles. The background was measured without the artificial fog and ranged 8-20 cpm. The neck-fan employed here is a typical personal cooling device ("W Fan", Spice, Nagoya Japan) whose slow wind runs approximately $2.5 \mathrm{~m} / \mathrm{s}$ at the distance of $10 \mathrm{~cm}$.

Data comparisons and statistical analyses

All the experiments were performed 3 times by one subject, the corresponding author, and average and standard deviations were obtained. The differences of front and upward droplet amounts were statistically compared using Student's T-test, one-tailed. Simultaneous measurements of three distinct points were achieved with three dust indicators to enable accurate comparisons. To compare the exhalations from mouth and nose, two dust indicators were set $20 \mathrm{~cm}$ away of face, as being isolated each other with a flexible plastic plate. 


\section{Abbreviations}

cpm count per a minute

\section{Declarations}

Acknowledgements

We would acknowledge Mr. Shiro Kataoka, All-Japan Karaoke Industrialist Associations for personal communications on epidemiological data in Karaoke businesses.

Authors' contributions

All the authors (TY, ES, NI and TN) had full access to all of the data in the study and take responsibility for the integrity of the data and the accuracy of the data analysis.

All the authors (TY, ES, NI and TN) had reviewed and approved the manuscript.

Concept and design: TY

Acquisition, analysis, or interpretation of data: All the authors (TY, ES, NI and TN).

Drafting of the manuscript: TY

Critical revision of the manuscript for important intellectual content: TY, ES.

Statistical analysis: TY, ES.

Administrative, technical, or material support: All the authors (TY, ES, NI and TN).

Supervision: TY, ES.

Declarations

Ethics approval and consent to participate.

It has been approved by the ethical committee at Fujita Health University (HM20-547) that this study fulfills relevant guidelines and regulations. The participant, who is the corresponding author himself, provided the consent for publication.

Availability of data and materials

The datasets used and analyzed during the current study are available from the corresponding author on reasonable request.

Competing interests 
The authors declare that they have no competing interest

Funding

This study was essentially funded on authors' private budgets.

\section{References}

1 Qian, H. et al. Indoor transmission of SARS-CoV-2. Indoor Air, doi:10.1111/ina.12766 (2020).

2 Chau, N. V. V. et al. Superspreading Event of SARS-CoV-2 Infection at a Bar, Ho Chi Minh City, Vietnam. Emerg Infect Dis 27, doi:10.3201/eid2701.203480 (2021).

3 Yong, S. E. F. et al. Connecting clusters of COVID-19: an epidemiological and serological investigation. Lancet Infect Dis 20, 809-815, doi:10.1016/S1473-3099(20)30273-5 (2020).

$4 \quad$ Althouse, B. M. et al. Superspreading events in the transmission dynamics of SARS-CoV-2: Opportunities for interventions and control. PLoS Bio/ 18, e3000897, doi:10.1371/journal.pbio.3000897 (2020).

5 Wang, L. et al. Inference of person-to-person transmission of COVID-19 reveals hidden superspreading events during the early outbreak phase. Nat Commun 11, 5006, doi:10.1038/s41467-02018836-4 (2020).

6 Zhang, Y., Li, Y., Wang, L., Li, M. \& Zhou, X. Evaluating Transmission Heterogeneity and SuperSpreading Event of COVID-19 in a Metropolis of China. Int J Environ Res Public Health 17, doi:10.3390/ijerph17103705 (2020).

7 Lau, M. S. Y. et al. Characterizing superspreading events and age-specific infectiousness of SARS-CoV-2 transmission in Georgia, USA. Proc Natl Acad Sci U S A 117, 22430-22435, doi:10.1073/pnas.2011802117 (2020).

8 Endo, A., Centre for the Mathematical Modelling of Infectious Diseases, C.-W. G., Abbott, S., Kucharski, A. J. \& Funk, S. Estimating the overdispersion in COVID-19 transmission using outbreak sizes outside China. Wellcome Open Res 5, 67, doi:10.12688/wellcomeopenres.15842.3 (2020).

9 Lloyd-Smith, J. O., Schreiber, S. J., Kopp, P. E. \& Getz, W. M. Superspreading and the effect of individual variation on disease emergence. Nature 438, 355-359, doi:10.1038/nature04153 (2005).

10 Kupferschmidt, K. Case clustering emerges as key pandemic puzzle. Science 368, 808-809, doi:10.1126/science.368.6493.808 (2020).

11 Lu, J. et al. COVID-19 Outbreak Associated with Air Conditioning in Restaurant, Guangzhou, China, 2020. Emerg Infect Dis 26, 1628-1631, doi:10.3201/eid2607.200764 (2020). 
12 Shen, Y. et al. Community Outbreak Investigation of SARS-CoV-2 Transmission Among Bus Riders in Eastern China. JAMA Intern Med, doi:10.1001/jamainternmed.2020.5225 (2020).

13 Martyr, K. https://www.dw.com/en/german-church-at-the-center-of-over-100-coronaviruscases/a-53804687 (Deutsche Welle, 2020).

14 Network, C. G. T. https://news.cgtn.com/news/2020-2010-2011/Cluster-of-infections-amongtheater-troupe-in-Japan-s-Saitama-UuHt2021Fooda/index.html (2020).

15 Harrichandra, A., lerardi, A. M. \& Pavilonis, B. An estimation of airborne SARS-CoV-2 infection transmission risk in New York City nail salons. Toxicol Ind Health, 748233720964650, doi:10.1177/0748233720964650 (2020).

16 Peter de Man, S. P., David S Y Ong, Norbert Vaessen, Gerard van Nielen, Johannes G M Koeleman. Outbreak of Coronavirus Disease 2019 (COVID-19) in a Nursing Home Associated With Aerosol Transmission as a Result of Inadequate Ventilation. Clin Infect Dis (2020).

17 Adam, D. C. et al. Clustering and superspreading potential of SARS-CoV-2 infections in Hong Kong. Nat Med 26, 1714-1719, doi:10.1038/s41591-020-1092-0 (2020).

18 Ma, J. et al. COVID-19 patients in earlier stages exhaled millions of SARS-CoV-2 per hour. Clin Infect Dis, doi:10.1093/cid/ciaa1283 (2020).

19 Liu, Y. et al. Aerodynamic analysis of SARS-CoV-2 in two Wuhan hospitals. Nature 582, 557-560, doi:10.1038/s41586-020-2271-3 (2020).

20 Morawska, L. \& Cao, J. Airborne transmission of SARS-CoV-2: The world should face the reality. Environ Int 139, 105730, doi:10.1016/j.envint.2020.105730 (2020).

21 Riediker, M. \& Tsai, D. H. Estimation of Viral Aerosol Emissions From Simulated Individuals With Asymptomatic to Moderate Coronavirus Disease 2019. JAMA Netw Open 3, e2013807, doi:10.1001/jamanetworkopen.2020.13807 (2020).

22 Yan, J. et al. Infectious virus in exhaled breath of symptomatic seasonal influenza cases from a college community. Proc Natl Acad Sci U S A 115, 1081-1086, doi:10.1073/pnas.1716561115 (2018).

23 van Doremalen, N. et al. Aerosol and Surface Stability of SARS-CoV-2 as Compared with SARSCoV-1. N Engl J Med 382, 1564-1567, doi:10.1056/NEJMc2004973 (2020).

24 Schuit, M. et al. The Influence of Simulated Sunlight on the Inactivation of Influenza Virus in Aerosols. J Infect Dis 221, 372-378, doi:10.1093/infdis/jiz582 (2020).

25 Prentiss, M., Chu, A. \& Berggren, K. K. Superspreading Events Without Superspreaders: Using High Attack Rate Events to Estimate No for Airborne Transmission of COVID-19 (Cold Spring Harbor Laboratory, 
2020).

26 Asadi, S. et al. Aerosol emission and superemission during human speech increase with voice loudness. Sci Rep 9, 2348, doi:10.1038/s41598-019-38808-z (2019).

27 Loudon, R. G. \& Roberts, R. M. Singing and the dissemination of tuberculosis. Am Rev Respir Dis 98, 297-300, doi:10.1164/arrd.1968.98.2.297 (1968).

28 Johnson, G. R. et al. Modality of human expired aerosol size distributions. Journal of Aerosol Science 42, 839-851 (2011).

29 Anchordoqui, L. A. \& Chudnovsky, E. M. A Physicist View of COVID-19 Airborne Infection through Convective Airflow in Indoor Spaces. SciMedicine Journal 2, 68-72 (2020).

30 Netz, R. R. Mechanisms of Airborne Infection via Evaporating and Sedimenting Droplets Produced by Speaking. J Phys Chem B 124, 7093-7101, doi:10.1021/acs.jpcb.0c05229 (2020).

31 Seyran, M. et al. The structural basis of accelerated host cell entry by SARS-CoV-2dagger. FEBS J, doi:10.1111/febs.15651 (2020).

32 Azzi, L. et al. Saliva is a reliable tool to detect SARS-CoV-2. J Infect 81, e45-e50, doi:10.1016/j.jinf.2020.04.005 (2020).

33 Sakaguchi, W. et al. Existence of SARS-CoV-2 Entry Molecules in the Oral Cavity. Int J Mol Sci 21, doi:10.3390/ijms21176000 (2020).

34 Sueki, A. et al. Evaluation of saliva as diagnostic materials for influenza virus infection by PCRbased assays. Clin Chim Acta 453, 71-74, doi:10.1016/j.cca.2015.12.006 (2016).

35 Milton, D. K., Fabian, M. P., Cowling, B. J., Grantham, M. L. \& McDevitt, J. J. Influenza virus aerosols in human exhaled breath: particle size, culturability, and effect of surgical masks. PLoS Pathog 9, e1003205, doi:10.1371/journal.ppat.1003205 (2013).

36 Gupta, J. K., Lin, C.-H. \& Chen, Q. Characterizing exhaled airflow from breathing and talking. Indoor Air 20, 31-39 (2009).

37 Eslami, H. \& Jalili, M. The role of environmental factors to transmission of SARS-CoV-2 (COVID19). AMB Express 10, 92, doi:10.1186/s13568-020-01028-0 (2020).

38 Abkarian, M., Mendez, S., Xue, N., Yang, F. \& Stone, H. A. Speech can produce jet-like transport relevant to asymptomatic spreading of virus. Proc Natl Acad Sci U S A 117, 25237-25245, doi:10.1073/pnas.2012156117 (2020).

39 Chen, Q. Can we migrate COVID-19 spreading risk? Front Environ Sci Eng 15, 35, doi:10.1007/s11783-020-1328-8 (2021). 
40 Rundfunk, B. https://www.br-klassik.de/aktuell/news-kritik/chor-corona-chorstudie-singeninfektionsgefahr-br-muenchen-erlangen-100.html?

fbclid=IwAR101ScmTVmtdQucbcG108kH107FFuIXtZWIDJUttrKU102hVnGFgwoARJuYeNFFgs108 (Bayerishcer Rundfunk, 2020).

41 Sara Kunkel, E. K., Aleksandra Arcipowska, Francesco Mariottini, Bogdan Atanasiu. in Buildings Performance Institute Europe https://www.bpie.eu/wpcontent/uploads/2015/2010/BPIE_executive_briefing_IAQ2015.pdf (2015).

42 Japanese, G. http://www.bungo.or.jp/t-shikai/linksigoto/kikaikanki.pdf http://www.iibh.org/kijun/pdf/BCinJ_Part_D_1910.pdf (2003).

43 Japan, C. b. c. o. https://www.cbcj.catholic.jp/2020/2011/2010/21468/ (2020).

44 NHK. in Karaoke Boxes in Japan Japanology https://www.youtube.com/watch?v=_ttZPMvSEM (2018).

45 Cao, G. et al. Protected zone ventilation and reduced personal exposure to airborne crossinfection. Indoor Air 25, 307-319, doi:10.1111/ina.12142 (2015).

\section{Figures}
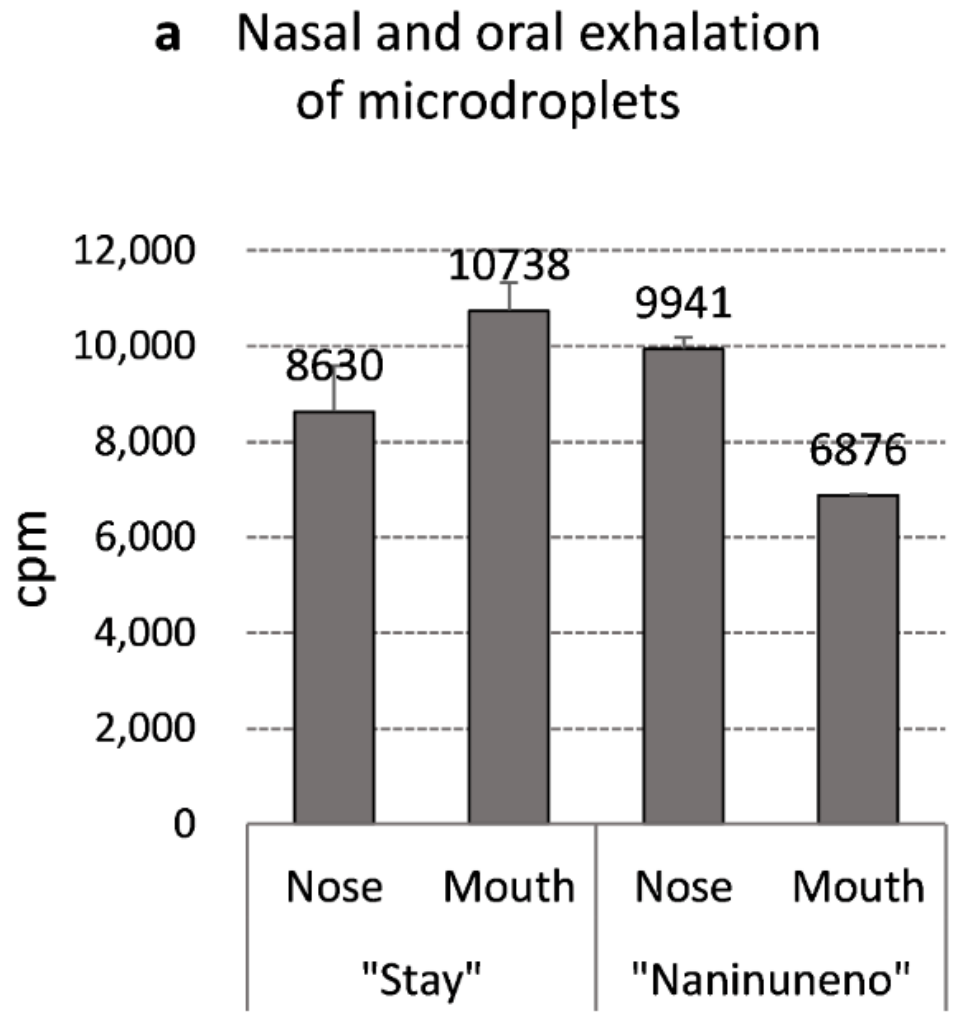

\section{b Height distributions of microdroplets}

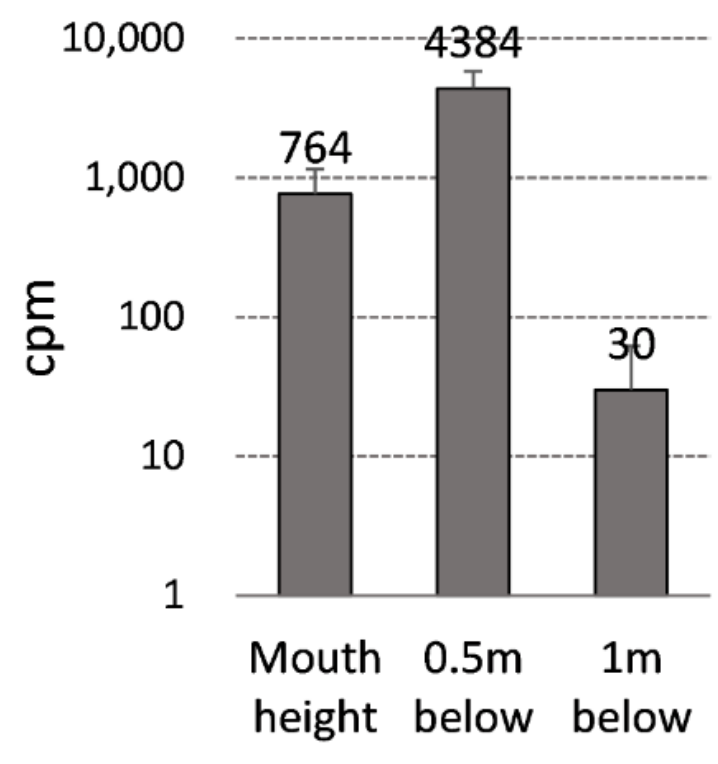




\section{Figure 1}

Microdroplets are exhaled predominantly downward through mouth and nose. A subject inhaled the artificial microdroplets from a fog machine and spoke "Stay healthy" or "na-ni-nu-ne-no" (Figure 1a) 3 times. Averages and standard deviations are depicted. A natural distribution of the exhaled microdroplets were quantified on digital dust meters as the accumulated average of $10 \mathrm{sec}$. a, The exhaled microdroplets, from nose and mouth were compared at $20 \mathrm{~cm}$ away, simultaneously. Nose and mouth were isolated with a soft plastic plate between then. $b$, The exhaled microdroplets were quantified at 3 indicated heights at $50 \mathrm{~cm}$ front, simultaneously.

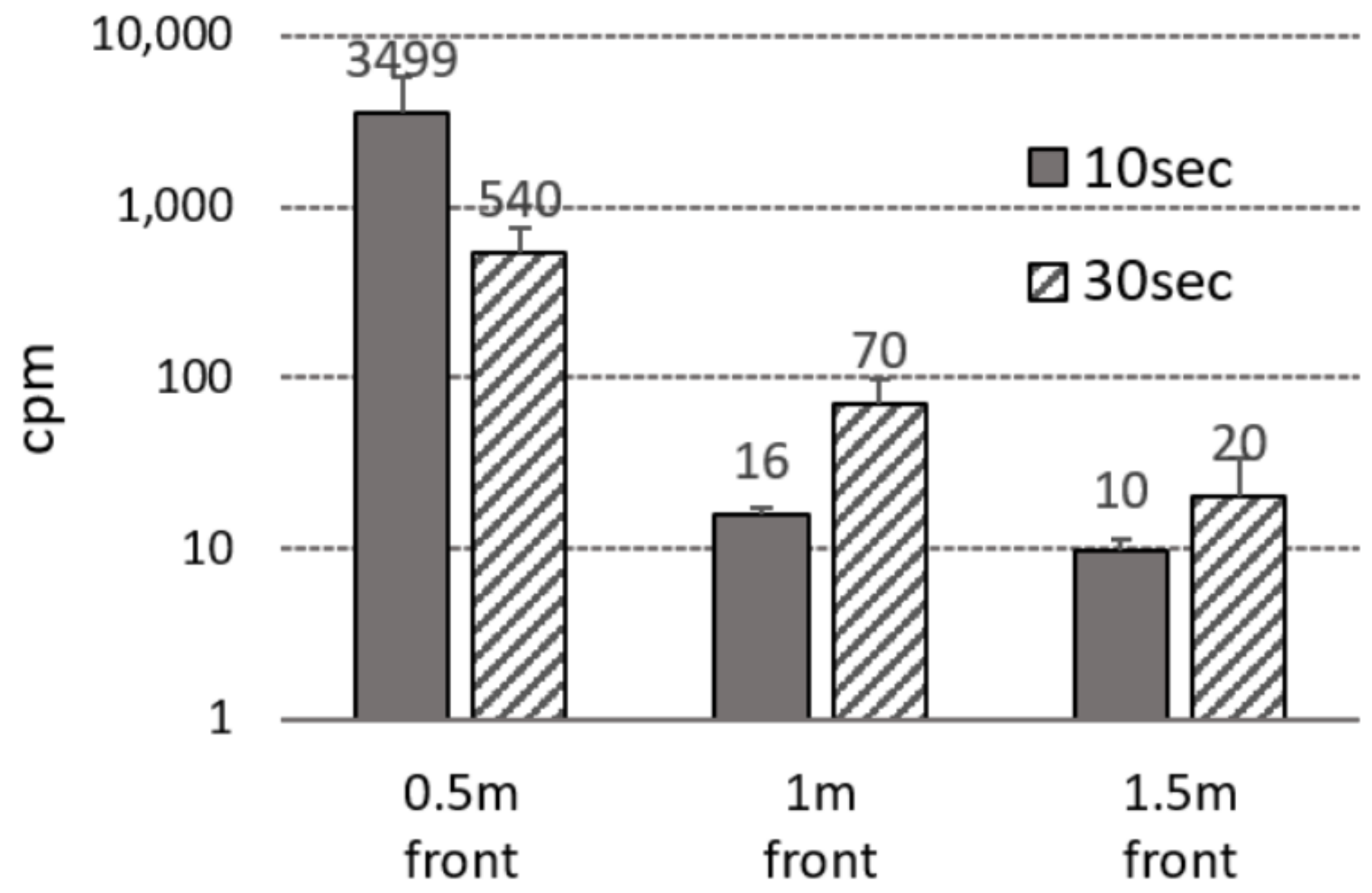

Figure 2

Microdroplets travel beyond $1.5 \mathrm{~m}$ within $30 \mathrm{sec}$. The same microdroplets were inhaled and exhaled by speaking "Stay Healthy". Experiments were repeated 3 times, and averages and standard deviations are depicted. The microdroplets were quantified at 3 indicated distances at $50 \mathrm{~cm}$ below of mouth, simultaneously. The accumulated average counts of 10 and $30 \mathrm{sec}$ were depicted. 


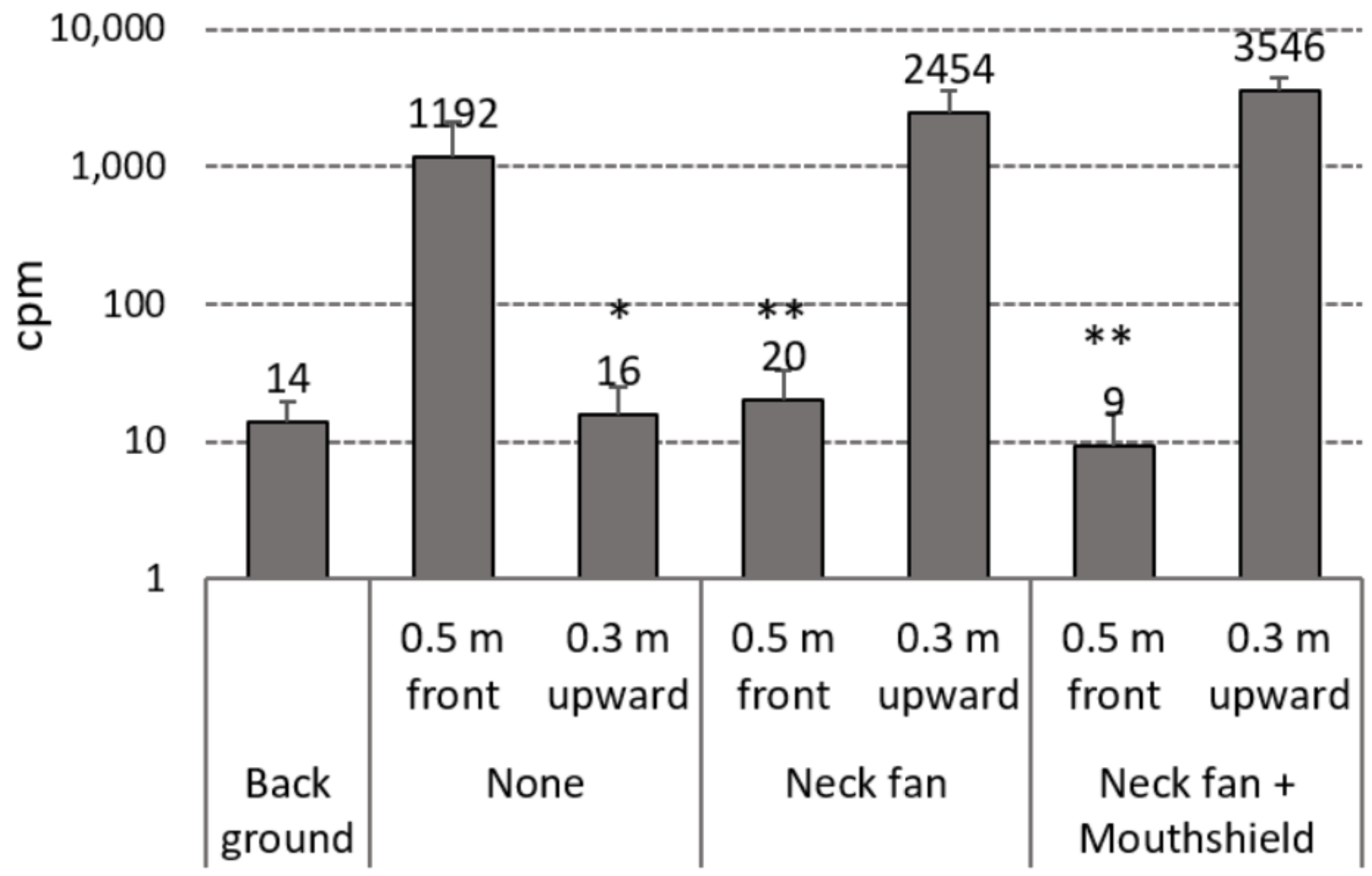

Figure 3

The effect of an air flow and mouth shield on microdroplets distributions The background was taken without any fog. The microdroplets were exhaled in same way as Figure 1 and 2, except applying safety devices. The " $50 \mathrm{~cm}$ front" is at the same height of mouth and " $30 \mathrm{~cm}$ up" is straight up from the mouth position. The data were accumulated averages of $10 \mathrm{sec}$ with the indicated devices. The differences were statistically analyzed with Student's T-test $(n=3$, one-tailed). P values are * $:<0.05 ; * \star:<0.01$. 


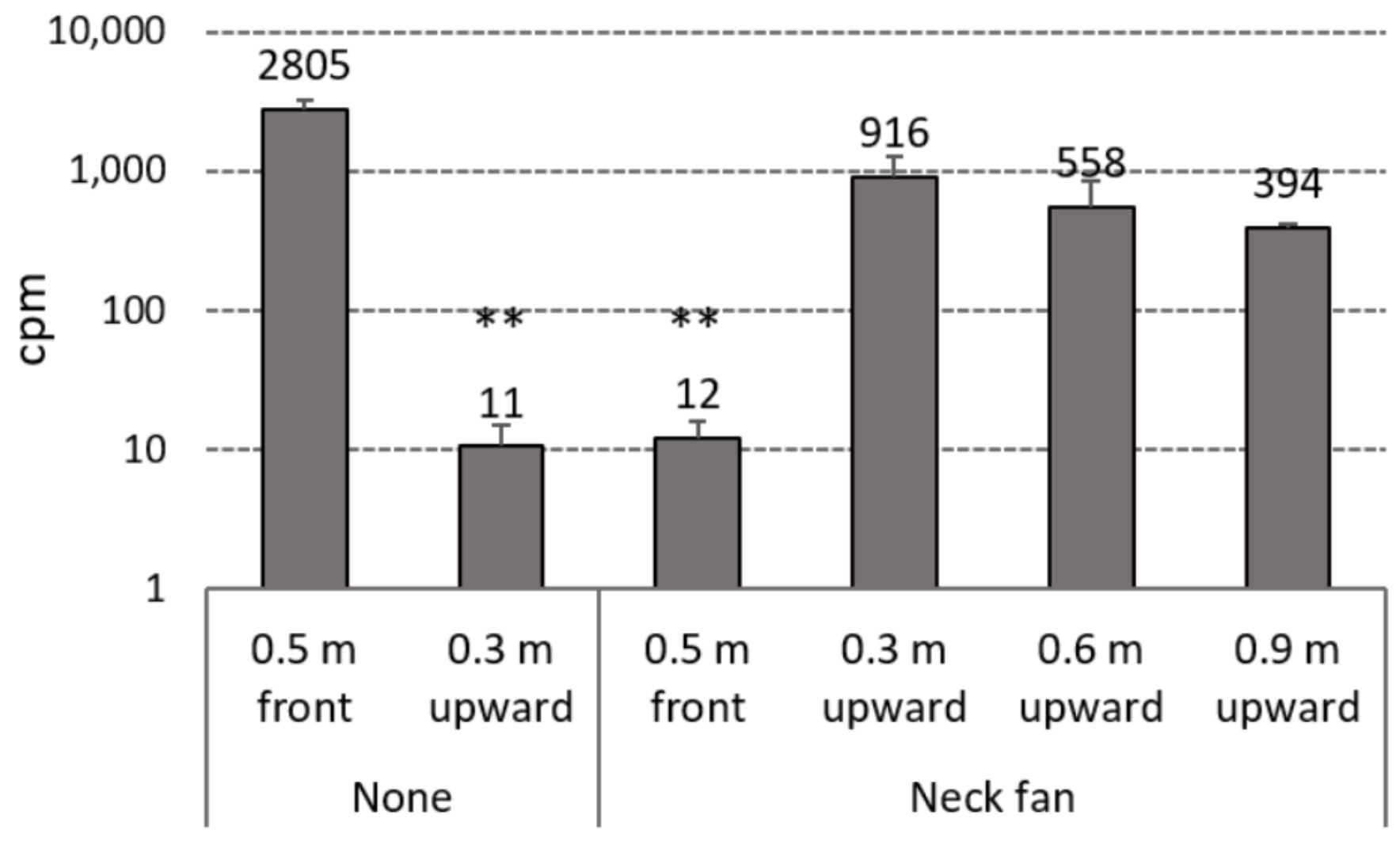

Figure 4

The reaching heights of microdroplets by the minimal air flow of a personal fan. The microdroplets were exhaled in same way with or without a neck-fan alone, and were quantified at the indicated positions, The data were accumulated and averaged for $30 \mathrm{sec}$. The differences were statistically analyzed with Student's T-test $(n=3$, one-tailed). $P$ value is $* *:<0.01$.

\section{Supplementary Files}

This is a list of supplementary files associated with this preprint. Click to download.

- YoshidasuplFeb11.pptx 